\title{
Lattice study of $J / \psi-\phi$ scattering at low energies to search for narrow resonance
}

\section{Sho Ozaki*}

Theoretical Research Division, Nishina Center, RIKEN

Institute of Physics and Applied Physics, Yonsei University

E-mail: shodrcnp.osaka-u.ac.jp

\section{Shoichi Sasaki}

Department of Physics, Tohoku University

Theoretical Research Division, Nishina Center, RIKEN

E-mail: ssasaki@nucl.phys.tohoku.ac.jp

\begin{abstract}
We develop an approach based on the Lüshcer's finite size method under twisted boundary conditions. It allows us to evaluate the scattering phase shifts at any small value of the scattering momentum even in a single finite volume. In this study, as a simple example, we demonstrate our method for calculating both $S$-wave and $P$-wave $J / \psi-\phi$ scattering phase shifts to search for $Y$ (4140) resonance using 2+1 flavor PACS-CS gauge configuration with partially twisted boundary conditions.
\end{abstract}

The 30th International Symposium on Lattice Field Theory

June 24 - 29, 2012

Cairns, Australia

\footnotetext{
${ }^{*}$ Speaker.
} 


\section{Introduction}

Recently, many charmonium- and bottomonium-like resonances which are so-called $X Y Z$ [1] and $Y_{b} Z_{b}$ [2, 3] resonances have been reported in several high-energy experiments. Among them, some resonances are observed near two-particle thresholds, and their widths are quite narrow although masses of such resonances are much higher than the $D \bar{D}$ threshold. In year 2009, the $Y(4140)$ resonance has been reported by the CDF collaboration [4]. The signal is observed in the invariant mass of the $J / \psi \phi$ pairs of the decay $B^{+} \rightarrow J / \psi \phi K^{+}$from $p \bar{p}$ collisions at $\sqrt{s}=1.96$ $\mathrm{TeV}$. Observed mass and width are $4143.4_{-3.0}^{+2.9} \pm 0.6 \mathrm{MeV}$ and $15.3_{-6.1}^{-10.4} \pm 2.5 \mathrm{MeV}$, respectively. In particular, the width is very narrow as compared to typical hadron resonances. This observation suggests that the $Y(4140)$ scarcely couples to open charm channels such as $D \bar{D}$ and also $D_{s}^{+} D_{s}^{-}$. On the other hand, the Belle and LHCb collaborations have not yet found the $Y(4140)$ in their experiments [5, 6]. Although the $Y(4140)$ might have very interesting properties, its existence is still controversial experimentally. In this study, we investigate the low-energy $J / \psi-\phi$ scattering using lattice QCD and try to give a new insight of the $Y(4140)$ resonance.

In order to investigate the low-energy hadron-hadron scattering and also search for narrow resonance, we apply an idea of twisted boundary conditions to the Lüscher's finite size method as originally proposed by Bedaque [7]. Since the twisted boundary conditions allow us to evaluate scattering phase shifts of the two-particle system at any small value of the relative momentum even in a finite box, detailed information of the low-energy interaction, which is described by the lower partial-wave phase shifts, such as the $S$-wave and $P$-wave phase shifts, at low energies, can be obtained through extended finite size method for the twisted boundary conditions.

\section{Formalism}

First of all, we introduce the twisted boundary condition, which is given as [7]

$$
\psi\left(x+L \vec{\varepsilon}_{i}\right)=e^{i \theta_{i}} \psi(x) \quad(i=x, y, z),
$$

where the angle variable $\theta$ is called the twist angle. $\psi$ stands for either elementary or composite field operators. Eq. 2.1) provides the quantization condition on the three-momentum for the noninteracting case:

$$
p_{i}=\frac{2 \pi}{L}\left(n_{i}+\frac{\theta_{i}}{2 \pi}\right) \quad(i=x, y, z) .
$$

Taking an advantage of the twisted boundary condition, we can, in principle, access finite, but arbitrary small momenta even in a single finite box through the variation of the twist angle. This technique is widely used in several lattice analyses [8, 9, 10].

Next, we consider the finite size formula with the twisted boundary conditions. In this study, we only consider the center of mass (CM) system with zero total momentum $\vec{P}=0$. When $\vec{\theta}=0$, the cubic symmetry is surely satisfied in the system, and we then can apply the original Lüscher's finite size formula [11]

$$
\cot \delta_{0}(k)=\frac{1}{\pi^{3 / 2} q} Z_{00}\left(1 ; q^{2}\right)
$$




\begin{tabular}{cccc}
\hline twist angle vector & $(0,0, \theta)$ & $(\theta, \theta, 0)$ & $(\theta, \theta, \theta)$ \\
symmetry & $C_{4 v}$ & $C_{2 v}$ & $C_{3 v}$ \\
\hline $\mathscr{M}_{S S}^{\vec{\theta}}$ & $w_{00}$ & $w_{00}$ & $w_{00}$ \\
$\mathscr{M}_{S P}^{\vec{\theta}}$ & $i \sqrt{3} w_{10}$ & $i \sqrt{6} w_{11}$ & $i 3 w_{10}$ \\
$\mathscr{M}_{P P}^{\vec{\theta}}$ & $w_{00}+2 w_{20}$ & $w_{00}-w_{20}-i \sqrt{6} w_{22}$ & $w_{00}-i 2 \sqrt{6} \omega_{22}$ \\
\hline
\end{tabular}

Table 1: For simplicity, an irrelevant phase factor is omitted in the definition of $\mathscr{M}_{S P}$. The explicit expression of $w_{l m}$ is given in the text.

Here $Z_{00}\left(s ; q^{2}\right)$ is the generalized zeta-function, which is defined as

$$
Z_{l m}\left(s ; q^{2}\right)=\sum_{\vec{n} \in \mathbb{Z}^{3}} \frac{\mathscr{Y}_{l m}(\vec{n})}{\left(\vec{n}^{2}-q^{2}\right)^{s}}, \quad q^{2}=\left(\frac{L k}{2 \pi}\right)^{2} .
$$

with $\mathscr{Y}_{l m}(\vec{n})=|\vec{n}|^{l} Y_{l m}\left(\Omega_{n}\right)$, and $k$ is the relative momentum. In the case of twisted boundary conditions, where the lowest Fourier mode, $\vec{n}=0$, still can receive non-zero momentum, the cubic symmetry is broken into its subgroup symmetry in the reciprocal lattice. For instance, when we take a twist angle vector $\vec{\theta}=(0,0, \theta)$, the lattice grid in momentum space is defined by vectors, $\vec{r}=\vec{n}+\vec{d}$ where $\vec{n} \in \mathbb{Z}^{3}$ and $\vec{d}=(0,0, \theta / 2 \pi)$. In $0<\theta<\pi$, the $z$-component of $\vec{r}$ is shifted by $d_{z}=\theta / 2 \pi$. This implies that the symmetry of the reciprocal lattice can be described by $C_{4 v}$ group. Similarly, when $\vec{\theta}=(\theta, \theta, 0)$, the symmetry can be described by $C_{2 v}$ group. When $\vec{\theta}=(\theta, \theta, \theta)$, the symmetry can be described by $C_{3 v}$ group. An important point of these symmetries $C_{n v}$ is that they do not have parity symmetry. As a consequence, even- $l$ and odd- $l$ partial waves do mix each other. Actually, these symmetries similarly appear in two-particle systems on moving frames for the case of two-particles with different masses. Such systems are well studied recently [12,13, 14, 15]. We can apply those knowledge to our twisted boundary cases.

In the case of twisted boundary conditions, the finite size formula for the $A_{1}$ sector is modified as the following determinant

$$
\left|\begin{array}{cc}
\cot \delta_{0}(k)-\mathscr{M}_{S S}^{\vec{\theta}}(q) & \mathscr{M}_{S P}^{\vec{\theta}}(q) \\
\mathscr{M}_{S P}^{\vec{\theta}}(q)^{*} & \cot \delta_{1}(k)-\mathscr{M}_{P P}^{\vec{\theta}}(q)
\end{array}\right|=0,
$$

The matrix elements $\mathscr{M}_{S S}^{\vec{\theta}}, \mathscr{M}_{S P}^{\vec{\theta}}$ and $\mathscr{M}_{P P}^{\vec{\theta}}$ are given for three types of the twist angle vector in Table1, where we use the short-hand notation

$$
w_{l m}=\frac{1}{\pi^{3 / 2} \sqrt{2 l+1} q^{l+1}} Z_{l m}^{\vec{\theta}}\left(1 ; q^{2}\right)^{*} .
$$

The generalized zeta-function $Z_{l m}^{\vec{\theta}}\left(1 ; q^{2}\right)$ with the twist angle is defined as

$$
Z_{l m}^{\vec{\theta}}\left(s ; q^{2}\right)=\sum_{\vec{r} \in \Gamma_{\vec{\theta}}} \frac{\mathscr{Y}_{l m}(\vec{r})}{\left(\vec{r}^{2}-q^{2}\right)^{s}},
$$

with $\Gamma_{\vec{\theta}}=\left\{\vec{r} \mid \vec{r}=\vec{n}+\frac{\vec{\theta}}{2 \pi}, \vec{n} \in \mathbb{Z}^{3}\right\}$. This modified finite size formula shows the mixing of $S$ - and $P$ waves. However at $\theta=\pi$, the parity symmetry is restored, and the $S$-wave and $P$-wave phase shifts 
are disentangled owing to $\mathscr{M}_{S P}^{\vec{\theta}}=0$. In this case, Eq. (2.5) becomes the Rummukainen-Gottlieb type formula [16] with no Lorentz factor $\gamma$ (since $\gamma=1$ ). Here we neglect higher partial-wave contributions above $D$-wave $(l \geq 2)$.

\begin{tabular}{cccccc}
\hline$\beta$ & $a[\mathrm{fm}]$ & $L^{3} \times T$ & $\sim L a[\mathrm{fm}]$ & $c_{S W}$ & configs \\
\hline 1.9 & 0.0907 & $32^{3} \times 64$ & 2.9 & 1.715 & 198
\end{tabular}

Table 2: Parameters of $2+1$ flavor PACS-CS gauge configurations, generated using the Iwasaki gauge action and Wilson clover fermions, at $m_{\pi}=156(7) \mathrm{MeV}$ and $m_{K}=554(2) \mathrm{MeV}$ [17].

\section{Numerical results}

We apply our new approach to the low-energy $J / \psi-\phi$ scattering with partially twisted boundary conditions, where we impose twisted boundary conditions on the valence quark fields. If there exists the $Y(4140)$ as reported from the CDF collaboration, we would observe a narrow resonance in the low-energy $J / \psi-\phi$ system on the lattice. We perform simulations on $L^{3} \times T=32^{3} \times 64$ lattice with PACS-CS $N_{f}=2+1$ dynamical gauge configurations at the lightest pion mass, $m_{\pi}=156$ $\mathrm{MeV}$ [17]. We employ the Tsukuba type Relativistic Heavy Quark (RHQ) action for the charm quark [18, 19]. TABLE I and II show the summary of our simulation parameters. In order to measure the total energy of the $J / \psi-\phi$ system and then calculate the relative momentum $k$ in the CM system, we assign momentum $\vec{p}=\vec{\theta} / L$ to $J / \psi$ and the opposite one $-\vec{p}$ to $\phi$ by imposing the twisted boundary condition on the single charm (strange) quark inside of $J / \psi(\phi)$ with the twist angle vector $\vec{\theta}(-\vec{\theta})$. On the other hand, we do not impose the twisted boundary condition on antiquarks in $J / \psi$ and $\phi$, which obey the same boundary condition imposed on the sea strange quarks. Using the measured energy and relative momentum $k$, we use the extended finite size formulae (2.5) for the twisted boundary conditions.

Figure 1 shows results of $k \cot \delta_{0}$ calculated by using the Lüscher and Rummukainen-Gottlieb type formulae with specific twist angles $\vec{\theta}=(0,0,0),(0,0, \pi),(\pi, \pi, 0),(\pi, \pi, \pi)$ data, where there is no mixing of $S$ - and $P$-waves [7]. It is observed that $k \cot \delta_{0}$ monotonically increase as the momentum $k$ increases, and there is no non-analytic behavior in the range covered here. Therefore, we simply interpolate the four data points by the quadratic function of $k^{2}$ with the jackknife analysis. Combined with this information of the $S$-wave phase shift, we can evaluate $\cot \delta_{1}$ from the data of the relative momentum $k^{2}$ calculated with a twist angle vector, $\vec{\theta}=(\theta, \theta, \theta)$ through the formula:

$$
\cot \delta_{1}=w_{00}+2 \sqrt{6} \operatorname{Im}\left(w_{22}\right)+\frac{9 w_{10}^{2}}{\cot \delta_{0}-w_{00}} .
$$

Here we use five different angles, $\theta=1.84,1.92,2.14,2.35,2.56[\mathrm{rad}]$. The results are shown in the left panel of Fig. 2. Although a slight but monotonic increase is observed, the result of $k^{3} \cot \delta_{1}$ shows only very mild $k^{2}$-dependence. Near the threshold, $\theta$ becomes very small, and $\cot \delta_{0}-w_{00}$ is close to 0 . It is thus difficult to evaluate the second term of the right hand side of Eq. (3.1) precisely in this region.

We evaluate $k^{3} \cot \delta_{1}$ up to $k \sim 0.008$ from 0.02 , and then we extrapolate $k^{3} \cot \delta_{1}$ to the region $k^{2} \sim 0$ by using the linear fit as a function of $k^{2}$ within the jackknife analysis. From the inverse value 


\begin{tabular}{ccccccc}
\hline flavor & $\kappa$ & $\mathrm{v}$ & $r_{s}$ & $c_{B}$ & $c_{E}$ & Ref. \\
\hline strange & 0.13640 & 1.0 & 1.0 & 1.715 & 1.715 & [17 \\
charm & 0.10819 & 1.2153 & 1.2131 & 2.0268 & 1.7911 & 119 \\
\hline
\end{tabular}

Table 3: Parameters of clover fermion (strange) and the RHQ action (charm) used in this work.

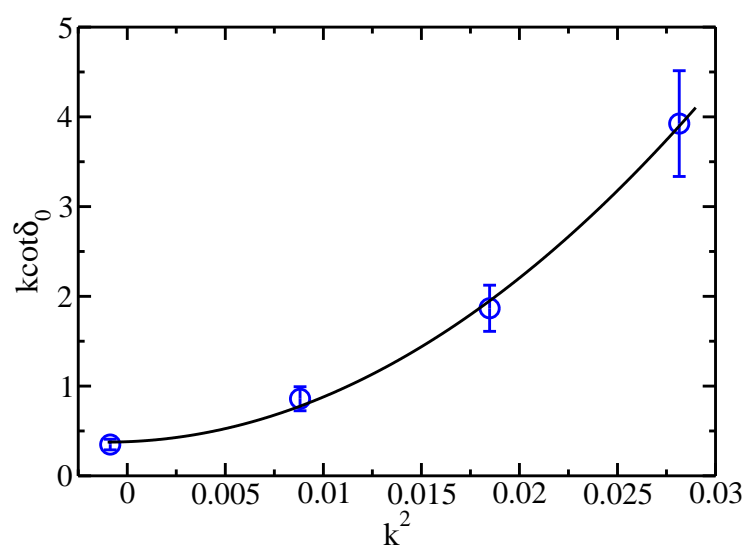

Figure 1: $k \cot \delta_{0}$ as a function of $k^{2}$ in lattice units. Open circles are calculated with twisted angles, $\vec{\theta}=$ $(0,0,0),(0,0, \pi),(\pi, \pi, 0)$, and $(\pi, \pi, \pi)$. A solid curve represents an interpolation of four data points using the effective range expansion up to the order of $k^{4}$.

of $k^{3} \cot \delta_{1}$ determined at $k=0$, we obtain the threshold parameter of the $P$-wave, the scattering volume $a_{1}$ as

$$
a_{1}=\left.\frac{\tan \delta_{1}}{k^{3}}\right|_{k=0}=0.0348 \pm 0.0081\left[\mathrm{fm}^{3}\right]
$$

The result of $a_{1}$ indicates that the $P$-wave interaction of the $J / \psi-\phi$ system is weakly attractive. Using the $P$-wave information, we evaluate $k \cot \delta_{0}$ at very low energies from the data calculated with $\vec{\theta}=(0,0, \theta)$ through Eq. (2.5) as

$$
\cot \delta_{0}=w_{00}+\frac{3 w_{10}^{2}}{\cot \delta_{1}-w_{00}-2 w_{20}} .
$$

Here we use three different angles, $\theta=1.44,2.16,2.88$ [rad]. The right panel of Fig. 2 shows the results of $k \cot \delta_{0}$. We have carried out correlated $\chi^{2}$ fits on all seven data displayed in the figure by using the form of the effective-range expansion:

$$
k \cot \delta_{0}(k)=\frac{1}{a_{0}}+\frac{1}{2} r_{0} k^{2}+\sum_{n=2}^{N} v_{n} k^{2 n}
$$

up to $N=4$, which corresponds to the order of $k^{8}$. From first three coefficients of the fitting function, we extract three low-energy scattering parameters, namely, the scattering length $a_{0}$, the 

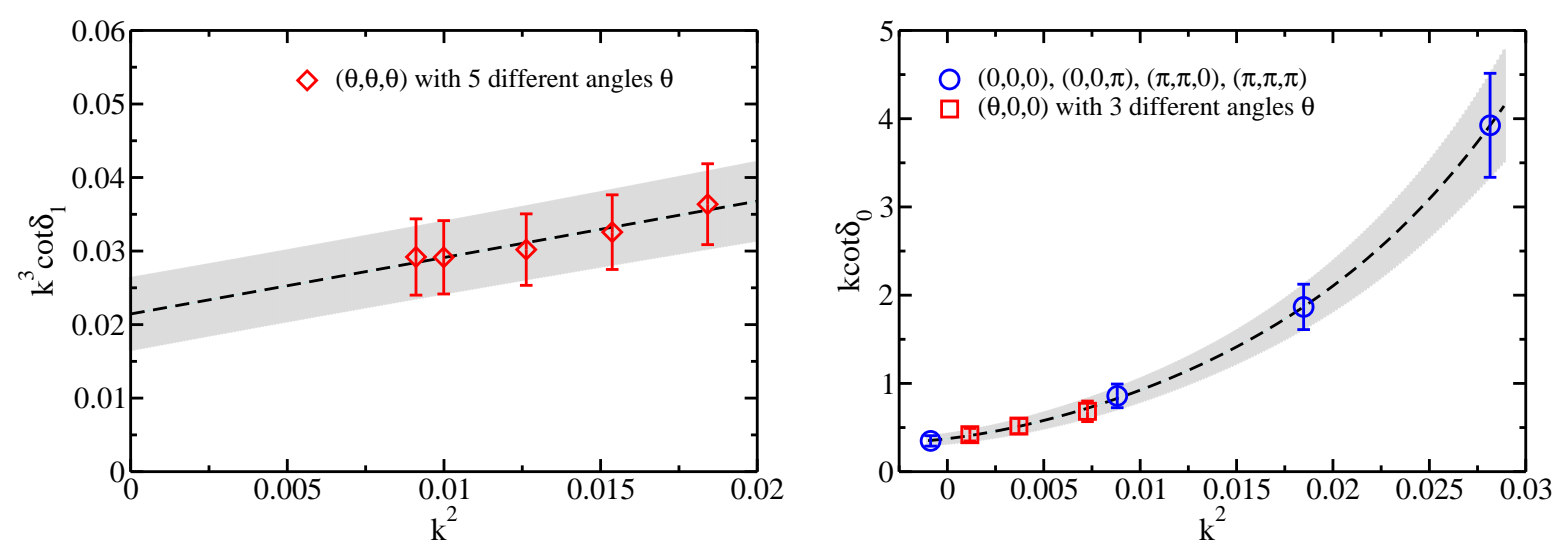

Figure 2: $k^{3} \cot \delta_{1}$ (left) and $k \cot \delta_{0}$ (right) as a function of $k^{2}$ in lattice units. In the left panel, open diamonds are calculated with a twist angle vector $\vec{\theta}=(\theta, \theta, \theta)$ with five different angles. In the right panel, there are same data appeared in Fig. 1 as open circles, while open squares stand for new data calculated with another twisted angle vector $\vec{\theta}=(0,0, \theta)$ with three different twist angles.

effective range $r_{0}$ and the effective volume $p r_{0}^{3}$ and then obtain

$$
\begin{aligned}
a_{0} & =0.242 \pm 0.041[\mathrm{fm}] \\
r_{0} & =4.71 \pm 1.73[\mathrm{fm}] \\
p r_{0}^{3} & =2.50 \pm 1.64\left[\mathrm{fm}^{3}\right] .
\end{aligned}
$$

The $S$-wave interaction is weakly attractive as well as the $P$-wave interaction. Figure 4 shows $S$ wave and $P$-wave scattering phase shifts of the low-energy $J / \psi-\phi$ system. We successfully obtain both $S$ - and $P$-wave phase shifts from our approach. Our results exhibit typical behaviors of weak attraction in the $S$-wave and $P$-wave phase shifts at low energies. Unfortunately, in both channels, there is no resonance structure associated with the $Y(4140)$ resonance against what we expected. Although the CDF experiment has reported evidence for a new resonance, $Y(4140)$ around a few $10 \mathrm{MeV}$ above the $J / \psi-\phi$ threshold, it seems that our results are consistent with null results in the Belle and LHCb experiments.

\section{Summary}

We develop an approach to investigate low-energy $J / \psi-\phi$ scattering with the finite size formula for the twisted boundary conditions. We successfully obtain the $P$-wave phase shift as well as the $S$-wave phase shift through the new formula, where the mixing of $S$ - and $P$-waves is taken into account properly. Our results show that although weak but attractive interaction appears in both $S$-wave and $P$-wave $J / \psi-\phi$ scatterings in low energy regions, there is no structure near the threshold contrary to what CDF has reported. It seems that our results are consistent with null results in the Belle and LHCb experiments. Instead of finding near-threshold and narrow resonance state, we succeed in extracting model independent low-energy parameters such as the scattering length, the effective range and the scattering volume from lattice QCD by using the finite size method with the partially twisted boundary conditions. 

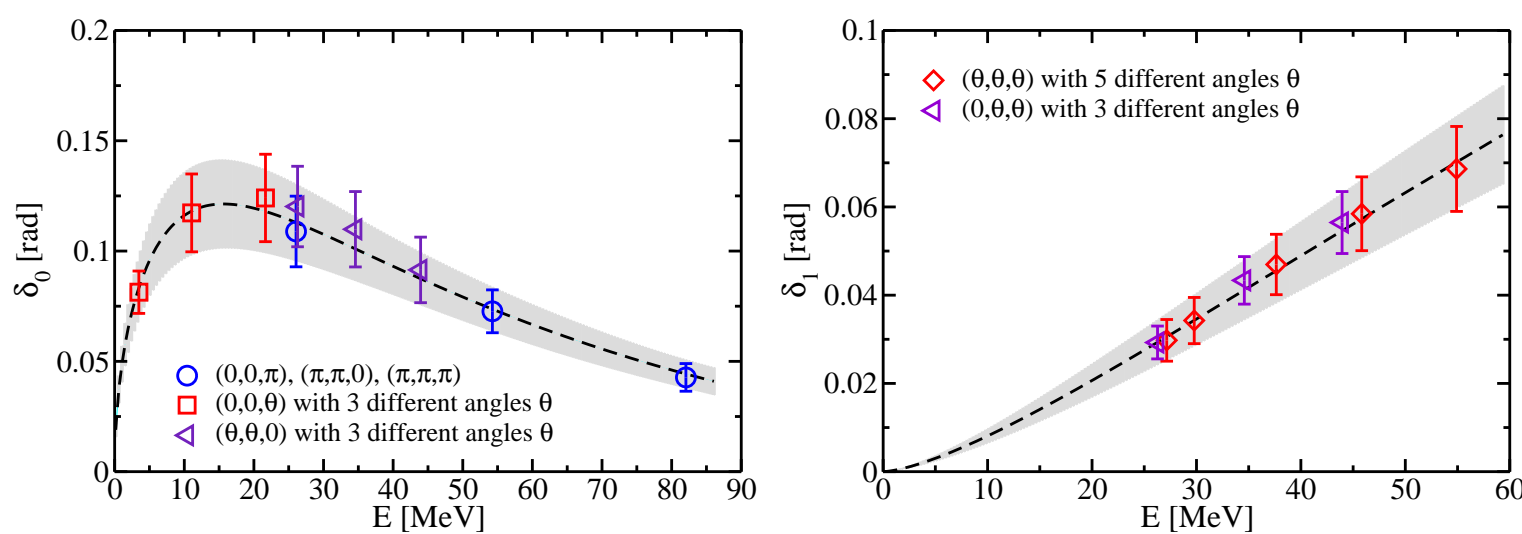

Figure 3: $S$-wave (left) and $P$-wave (right) scattering phase shifts as a function of the two particle energy $E$, which is measured from the $J / \psi-\phi$ threshold. In each panel, a solid line represents the fit result guided by the effective range expansion applied to all data points.

\section{Acknowledgments}

This work is supported by MEXT Grant-in-Aid for Scientific Research on Innovative Areas (No.2004:20105003) and JSPS Grant-in-Aid for Scientific Research (C) (No.23540284). Numerical calculations reported here were carried out on the T2K supercomputer at ITC, University of Tokyo and also at CCS, University of Tsukuba.

\section{References}

[1] N. Brambilla, S. Eidelman, B. K. Heltsley, R. Vogt, G. T. Bodwin, E. Eichten, A. D. Frawley and A. B. Meyer et al., Eur. Phys. J. C 71, 1534 (2011)

[2] K. F. Chen et al. [Belle Collaboration], Phys. Rev. Lett. 100, 112001 (2008)

[3] A. Bondar et al. [Belle Collaboration], Phys. Rev. Lett. 108, 122001 (2012)

[4] T. Aaltonen et al. [CDF Collaboration], Phys. Rev. Lett. 102, 242002 (2009)

[5] C. P. Shen et al. [Belle Collaboration], Phys. Rev. Lett. 104, 112004 (2010)

[6] R. Aaij et al. [LHCb Collaboration], arXiv:1202.5087 [hep-ex].

[7] P. F. Bedaque, Phys. Lett. B 593, 82 (2004)

[8] G. M. de Divitiis, R. Petronzio and N. Tantalo, Phys. Lett. B 595, 408 (2004)

[9] P. A. Boyle et al., JHEP 0807, 112 (2008)

[10] C. H. Kim and C. T. Sachrajda, Phys. Rev. D 81, 114506 (2010)

[11] M. Lüscher, Nucl. Phys. B 354, 531 (1991).

[12] Z. Fu, Phys. Rev. D 85, 014506 (2012)

[13] L. Leskovec and S. Prelovsek, Phys. Rev. D 85, 114507 (2012)

[14] M. Doring, U. G. Meissner, E. Oset and A. Rusetsky, arXiv:1205.4838 [hep-lat].

[15] M. Gockeler, R. Horsley, M. Lage, U. -G. Meissner, P. E. L. Rakow, A. Rusetsky, G. Schierholz and J. M. Zanotti, arXiv:1206.4141 [hep-lat]. 
[16] K. Rummukainen and S. A. Gottlieb, Nucl. Phys. B 450, 397 (1995).

[17] S. Aoki et al. [PACS-CS Collaboration], Phys. Rev. D 79, 034503 (2009)

[18] S. Aoki, Y. Kuramashi and S. -i. Tominaga, Prog. Theor. Phys. 109, 383 (2003).

[19] T. Kawanai and S. Sasaki, Phys. Rev. D 85, 091503 (2012). 